\title{
Distribución actual e importancia epidemiológica de las especies de triatominos (Reduviidae: Triatominae) en Colombia
}

Jorge A. Molina ${ }^{1}$, Luis E. Gualdrón ${ }^{2}$, Helena L. Brochero ${ }^{1}$, Víctor A. Olano ${ }^{1}$, Diana Barrios ${ }^{3}$, Felipe Guhl ${ }^{3}$

\author{
${ }^{1}$ Laboratorio de Entomología, Instituto Nacional de Salud, Bogotá, D.C., Colombia. \\ ${ }^{2}$ Laboratorio de Parasitología, Instituto Nacional de Salud, Bogotá, D.C., Colombia. \\ ${ }^{3}$ Centro de Investigaciones en Microbiología y Parasitologia Tropical, Universidad de los Andes, Bogotá, D.C., \\ Colombia.
}

Se presenta la distribución geográfica y la importancia epidemiológica en la transmisión de la enfermedad de Chagas de las 23 especies de triatominos presentes en Colombia, tomando como base los registros bibliográficos existentes en el país y las colecciones de referencia del Laboratorio de Entomología del Instituto Nacional de Salud y del Centro de Investigaciones en Microbiología y Parasitología Tropical de la Universidad de los Andes; además, se realiza un análisis de las condiciones ecológicas en las cuales han sido registrados triatominos en los países con fronteras con Colombia para poder establecer la presencia de posibles especies que aún no se han registrado en nuestro país. De esta manera, existe la posibilidad de tener en Colombia especies con hábitos silvestres como: Alberprosenia malheiroi, Belminus laportei y Cavernicola lenti, preentes todas ellas en los departamentos amazónicos; Triatoma nigromaculata en la Cordillera Oriental y con hábitos intradomiciliarios; Panstrongylus chinai con posibilidad de encontrarse en hábitats selváticos o en las viviendas de los colonos de la Amazonia debido a su atracción por la luz y Alberprosenia goyovargasi en hábitats silvestres de la frontera con Venezuela. Se resalta la importancia de la vigilancia entomológica a nivel nacional como estrategia para reforzar los estudios de triatominos silvestres en Colombia.

Palabras clave: Triatominae, distribución geográfica, enfermedad de Chagas, Colombia.

Present distribution and epidemiological importance of triatomine (Reduviidae: Triatominae) species in Colombia

We present the geographical distribution and epidemiological importance for Chagas' disease transmission of 23 triatomine species in Colombia, based on bibliographical reports available in the country and on reference collections housed in the Entomology Laboratory at the Instituto Nacional de Salud and the Tropical Microbiology and Parasitology Research Center at Universidad de los Andes. We also discuss the ecological conditions under wich triatomines in countries bordering Colombia have been reported, aimed at establishing the possible presence of species that have not been reported yet in our country. We, therefore, report possible presence in Colombia of species with wild habitats such as Alberprosenia malheiroi, Belminus laportei, and Cavernicola lenti, all of them located in the Amazon region; Triatoma nigromaculata, which shows intradomiciliary habits, in the Eastern mountain range; Panstrongylus chinai, probably located in wild habitats and also in settlers' dwellings in the Amazon region due to its attraction to light, and Alberprosenia goyovargasi, located in wild habitats in our border with Venezuela. The importance of national surveillance programs is highlighted as an aid strategy for the study of triatomine species in Colombia.

Key words: Triatomine, geographical distribution, Chagas disease, Colombia.

Correspondencia: volano@hemagogus.ins.gov.co

Recibido: 23/02/00; aceptado: 13/10/00 
Los triatominos son insectos grandes ( 5 a $44 \mathrm{~mm}$ ) con amplios abdómenes (1,9 a $15 \mathrm{~mm}$ ) y con cuatro alas, dos superiores con la parte anterior esclerotizada y la posterior membranosa, y dos inferiores con iguales rasgos. Tienen probócides recta dividida en tres partes, que se extiende ventralmente desde el ápice de la cabeza hasta la parte anterior del tórax cuando se mantiene en reposo. Las fases inmaduras (cinco estadios de ninfal) son terrestres y ocupan durante su desarrollo el mismo hábitat que los adultos. Machos, hembras y ninfas se alimentan exclusivamente de sangre de aves, mamíferos y reptiles y tienen actividad nocturna.

La subfamilia Triatominae presenta una gran diversidad de especies como producto de su adaptación a ecótopos artificiales y naturales que le han permitido la supervivencia y el aprovechamiento de los recursos existentes.

En Latinoamérica, las especies domiciliadas de la subfamilia han recibido el mayor interés debido a la importancia epidemiológica dentro del ciclo de transmisión de la enfermedad de Chagas, situación que ha relegado a segundo plano los estudios de los triatominos con hábitos silvestres.

Esta revisión toma como base dichos trabajos y los registros de triatominos silvestres existentes en los países fronterizos de Panamá, Venezuela, Brasil, Ecuador y Perú, con el propósito de presentar una aproximación real al estado del conocimiento actual sobre la subfamilia en nuestro país y señalar la posibilidad de aumentar la distribución y el número de especies de triatominos presentes en Colombia.

\section{Clasificación y especies alejadas de Colombia}

Siguiendo a Lent y Wygodzinsky (1), Schofield (2), Schofield (3), Carcavallo y colaboradores (4), Panzera (5), Carcavallo y colaboradores (6) y Moreno y colaboradores (7), la clasificación de las 130 especies de triatominos se presenta en el cuadro 1.

Con posterioridad al trabajo de Lent y Wygodzinsky (1), se crearon dos nuevos géneros: Hermanlentia (9) y Torrealbaia (10), y se revalidó el género Mepraia (11). Se describieron 16 especies nuevas: Alberprosenia malheiroi, Belminus pittieri, $B$. laportei, B. pittieri, Cavernicola lenti, Hermanlentia matsunoi, Mepraia gajardoi, Rhodnius colombiensis, R. stali, Triatoma bassolsae, $T$. brailovskyi, T. bolivari, T. carcavalloi, T. melanosoma, T. gomeznuñezi y Torrealbaia martinezi. Pasaron a ser sinónimos cuatro especies: $T$. funerea $=T$. melanosoma, $P$. salazari $=P$. arthuri, $P$. turpiali $=P$. chinai y $T$. gallardo $i=T$. patagonica (4).

\section{Distribución actual de los triatominos en Colombia}

De los 17 géneros de triatominos descritos, los siguientes no se pueden encontrar en nuestro país:

Bolbodera: exclusivo de la isla de Cuba; Dipetalogaster: exclusivo de México (Baja California); Linschosteus: exclusivo de India (Asia); Mepraia: exclusivo de Chile; Parabelminus: exclusivo del oriente de Brasil y Paratriatoma: exclusivo de México y Estados Unidos. Al descontar estos 6 géneros, simultáneamente estamos eliminando la presencia en Colombia de 12 especies.

De los restantes 11 géneros, se descuentan, también por ubicación geográfica, las siguientes especies: Belminus, una especie presente en Norte y Centroamérica; Psammolestes, 1 especie en el sur de Suramérica; Panstrongylus, 1 especie en el sur de Suramérica y Triatoma, 46 especies, siete del Viejo Mundo, 26 de Norte y Centroamérica, nueve del sur de Suramérica, tres de islas del Caribe y una del Viejo y Nuevo Mundo.

Así, de las 130 especies de triatominos descritas, se descartan 61, lo que determina que las restantes 69 se encuentran en Colombia y los países vecinos.

Las 23 especies descritas para Colombia son: Belminus herreri, Lent y Wygodzinsky, 1979; Belminus rugulosus, Stal, 1859; Cavernicola pilosa, Barber, 1937; Eratyrus cuspidatus, Stal, 1859; Eratyrus mucronatus, Stal, 1859; Microtriatoma trinidadensis, Lent, 1951; Panstrongylus geniculatus, Latreille, 1811; Panstrongylus humeralis, Usinger, 1939; Panstrongylus lignarius, Walker, 1873; Panstrongylus rufotuberculatus, Champion, 1899; Psammolestes arthuri, Pinto, 1926; Rhodnius 
Cuadro 1. Clasificación de las espesies de triatominos.

ORDEN: Hemiptera

SUBORDEN: Heteróptera

FAMILIA: Reduviidae

SUBFAMILIA: Triatominae

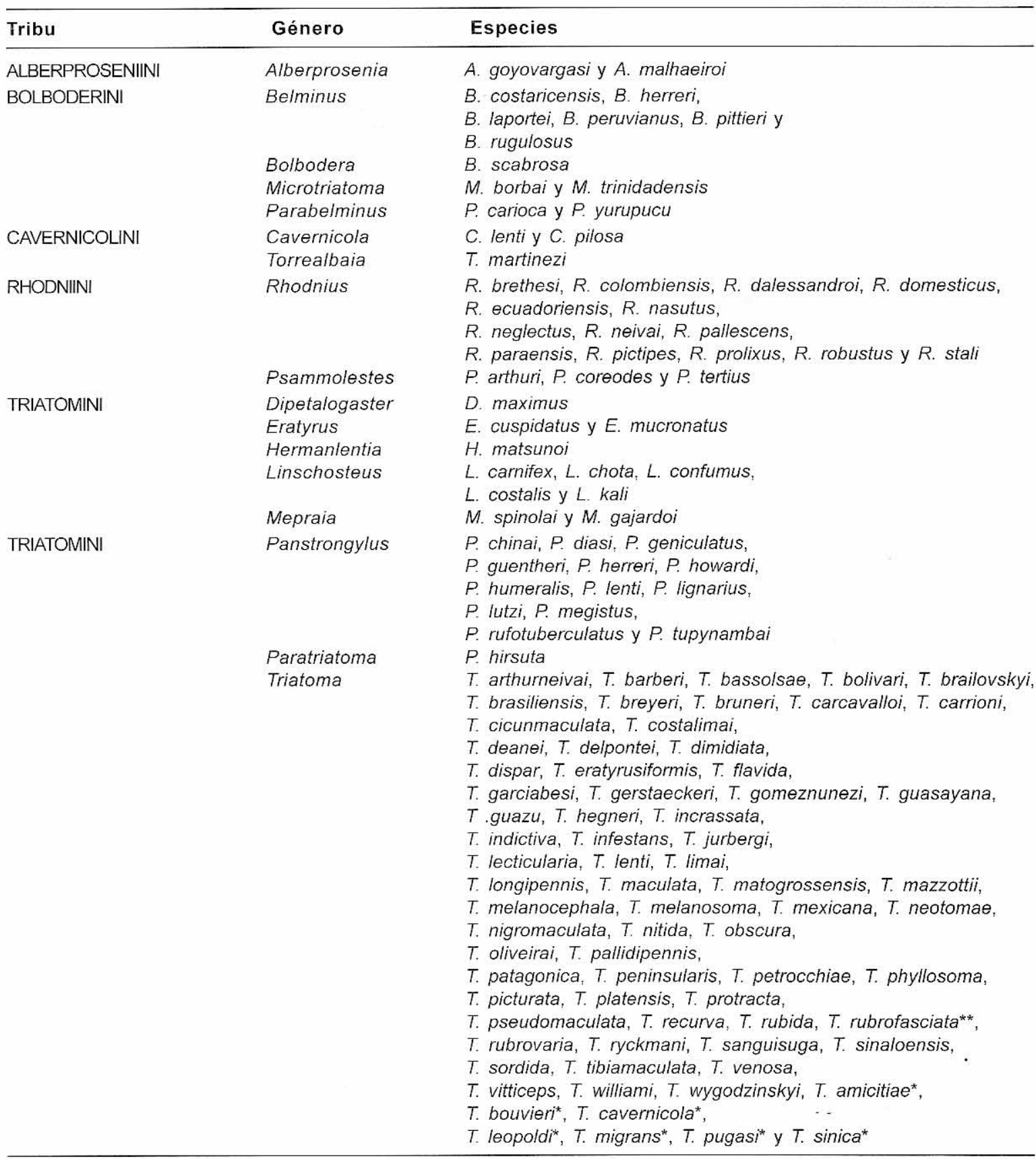

Dentro del género Triatoma, hay 7 especies $\left(^{*}\right)$ con registros en el Viejo Mundo, al igual que las cinco especies del género Linschosteus y una con registros en el Nuevo y Viejo Mundo $\left(^{* *}\right)(8)$. 
brethesi, Matta, 1919; Rhodnius colombiensis, Moreno, Galvao y Jurberg, 1999; Rhodnius dalessandroi, Carcavallo y Barreto, 1976; Rhodnius neivai, Lent, 1953; Rhodnius pallescens, Barber, 1932; Rhodnius pictipes, Stal, 1872; Rhodnius prolixus, Stal, 1859; Rhodnius robustus, Larrousse, 1927; Triatoma dimidiata, Latreille, 1811; Triatoma dispar, Lent, 1950; Triatoma maculata, Erichson, 1848 y Triatoma venosa, Stal, 1872.

La ubicación geográfica por departamento de las 23 especies de triatominos presentes en Colombia se resume en el cuadro 2 .

\section{Triatominos que se pueden encontrar en Colombia}

Con el ánimo de establecer las posibles especies de triatominos presentes en Colombia y que hasta el momento no han sido registradas, se abordó el conocimiento de las especies presentes en los países vecinos, la determinación de su distribución geográfica y las condiciones ecológicas en las que fueron registradas para relacionarlas con las presentes en Colombia. El análisis obtenido es el siguiente.

\section{Venezuela}

Para 1985 (12), se habían encontrado en Venezuela 18 especies; en 1993, Osuna y colaboradores aumentaron a 19 el número de las especies registradas con Belminus pittieri (4); en 1996, Valderrama y colaboradores registraron a Panstrongylus turpiali (sinónimo de Panstrongylus chinai) (4) y en 1998 Carcavallo y colaboradores a Torrealbaia martinezi (10), aumentando a 21 las especies registradas; si adicionamos el registro de Triatoma rubrofasciata, tenemos, entonces, que las 22 especies de triatominos para Venezuela son: Alberprosenia goyovargasi, Belminus rugulosus, B. pittieri, Cavernicola pilosa, Eratyrus cuspidatus, E. mucronatus, Microtriatoma trinidadensis, Panstrongylus chinai, P. geniculatus, $P$. lignarius, $P$. rufotuberculatus, $P$ sammolestes arthuri, Rhodnius brethesi, $R$. neivai, $R$. pictipes, $R$. prolixus, $R$. robustus, Torrealbaia martinezi, Triatoma dimidiata, T. maculata, T. nigromaculata y $T$. rubrofasciata.

De estas 22 especies, 16 están presentes en Colombia; las 6 que no se comparten son:
Alberprosenia goyovargasi, Belminus pittieri, Panstrongylus chinai, Torrealbaia martinezi, Triatoma nigromaculata y Triatoma rubrofasciata.

Alberprosenia goyovargasi: esta especie también es de distribución exclusiva en Venezuela y se ha capturado en el estado de Zulia en una sola oportunidad (13), lo que dificulta establecer un rango de distribución de la especie que facilite determinar su posible presencia en nuestro país. Sin embargo, debido a su localización fronteriza y su condición silvestre (asociada con la corteza de árboles muertos), se debe tener en cuenta para futuros estudios de triatominos silvestres en nuestro país.

Belminus pittieri: la información bibliográfica disponible sobre esta especie no es suficiente para establecer su posible presencia en nuestro país.

Panstrongylus chinai: esta especie se ha registrado en Ecuador, Perú y Venezuela. Tal distribución geográfica permite suponer su presencia en nuestro país. Su búsqueda debe centrarse en los departamentos de la Amazonia por tratarse de una especie con hábitos selváticos pero que, ocasionalmente, se puede encontrar en viviendas, atraída por la luz o en asocio con las aves de corral (1).

Torrealbaia martinezi: especie descrita a partir de una hembra capturada intradomiciliarmente en 1973 en el estado de Portuguesa (10). Debido a la presencia de la especie en un estado interior y por haber sido capturado un solo ejemplar, es difícil establecer su posible presencia en nuestro país.

Triatoma nigromaculata: especie con presencia exclusiva en Venezuela y que para 1959 (13) había sido registrada en los estados de Distrito Federal, Barinas, Mérida, Portuguesa y Yaracuy. Para 1985 (12), su distribución ya cubría los estados de Táchira, Aragua, Bolívar, Cojedes, Lara, Monagas, Sucre, Delta Amacuro y Trujillo. En 1994, Scorza, Solarte y Moreno (14) la registran como una especie intradomiciliar, natuialmente infectada con Trypanosoma cruzi, en la cordillera de los Andes en alturas superiores a los 1000 msnm y en viviendas en buenas condiciones. Este registro coloca al departamento de Norte de Santander (Colombia), municipios de Chinácota, Bochalema, 
Cuadro 2. Registro por departamentos de las especies presentes en Colombia; actualizado de Guhl F (94).

\begin{tabular}{|c|c|c|c|}
\hline Departamento & Especie & Flagelados & Municipio \\
\hline \multirow[t]{4}{*}{ Amazonas } & P. geniculatus & NE & Leticia (53), Tarapacá* \\
\hline & R. pictipes & $\mathrm{NR}$ & Puerto Nariño (53), Leticia (53) \\
\hline & R. prolixus & NE & Tarapacá* \\
\hline & R. robustus & $\mathrm{NE}$ & Leticia* \\
\hline \multirow[t]{11}{*}{ Antioquia } & B. rugulosus & NEG & San Carlos (31) \\
\hline & E. cuspidatus & NEG & San Carlos $(31,32)$ \\
\hline & E. mucronatus & NEG & San Carlos (31) \\
\hline & P. geniculatus & Tc & $\begin{array}{l}\text { Amalfi }(70) \text {, San Carlos }(31) \text {, Puerto Berrío }(31) \\
\text { Urabá }(61) \text {, Medellín }(44,75) \text {, Vegachí }(32,60,63,72)\end{array}$ \\
\hline & P. humeralis & NR & San Carlos $(31,83)$, Vegachí $(83)$, Puerto Berrío $(83,32)$ \\
\hline & P. rufotuberculatus & NR & San Carlos $(31,83)$, Vegachí $(83)$, Puerto Berrío $(83,32)$ \\
\hline & R. pallescens & Tc & San Carlos $(31,81)$, Vegachí $(31)$, Puerto Berrío, Turbo $(32,33)$ \\
\hline & R. prolixus & NE & $(32,60,72)$ \\
\hline & T. dimidiata & $\mathrm{NR}$ & $(32)$ \\
\hline & T. dispar & NR & Dabeiba $(67,32,61)$ \\
\hline & T. venosa & Tc & Puerto Nare $(26,32)$ \\
\hline \multirow[t]{5}{*}{ Arauca } & E. mucronatus & & $(45)$ \\
\hline & R. prolixus & NEG & $(45,60,72)$ \\
\hline & R. robustus & & (45) \\
\hline & T. dimidiata & & Arauca (45) \\
\hline & T. maculata & & Tame (45) \\
\hline Atlántico & T. maculata & NR & $(34,60,72)$ \\
\hline \multirow[t]{6}{*}{ Bolivar } & E. cuspidatus & NEG & (32) \\
\hline & P. geniculatus & NEG & $(32)$ \\
\hline & R. pallescens & NEG & $(32,60,63,72)$ \\
\hline & R. prolixus & NE & Las Boquillas* \\
\hline & R. robustus & NR & $(63)$ \\
\hline & T. maculata & NEG & $(32)$ \\
\hline \multirow[t]{8}{*}{ Boyacá } & E. cuspidatus & $\mathrm{NR}$ & San Pablo de Borbur $(40,41,60,63,72)$ \\
\hline & E. mucronatus & & Paez $(40,41)$ \\
\hline & P. geniculatus & & $\begin{array}{l}\text { Berbeo (41), Boavita (41), La Victoria (41), Maripí (41), Puerto } \\
\text { Boyacá (41), San Pablo de Borbur (41), San Eduardo (41), Santa } \\
\text { María (41), Zetaquirá (41), Soatá (41), Susacón }(40,41)\end{array}$ \\
\hline & P. rufotuberculatus & & San Pablo de Borbur $(41)$, Zetaquirá $(40,41)$ \\
\hline & R. pictipes & & Garagoa $(40,41)$ \\
\hline & R. prolixus & $\begin{array}{l}\text { Tc, Tr, } \\
\text { Tsp }\end{array}$ & $\begin{array}{l}\text { Almeida (41), Berbeo (41), Boavita (41), Campo Hermoso (41), } \\
\text { Chiquinquirá }(63,76) \text {, Chitaraque }(41) \text {, Chivor (41), Covarachía } \\
(41) \text {, Cubará }(41) \text {, Garagoa }(41,44,63) \text {, Guateque }(41,44,63 \text {, } \\
\text { 76), Guayatá }(41,63,76) \text {, Labranzagrande }(41) \text {, La Capilla (41), } \\
\text { La Uvita (41), Macanal }(41) \text {, Miraflores }(44,63) \text {, Moniquirá } \\
(41,44,63,76) \text {, Otanche }(41,63) \text {, Pachavita (41), Páez (41), } \\
\text { Pajarito (63), Pauna (63), Paya (41), Pisba (41), Puerto Boyacá } \\
\text { (41), Ramiriqui (63), Rondón (41), San Eduardo (41), San Luis } \\
\text { de Gaceno (41), San Mateo (41), Santa María (41), Sátiva } \\
\text { Norte (41), Soatá (41,44,63,76,80), Somondoco (41), Susacón } \\
\text { (41), Sutatenza (41), Tenza }(41) \text {, Tinjacá (63), Tipacoque (41), } \\
\text { Togui (41), Zetaquirá }(40,41,60,72)\end{array}$ \\
\hline & T. dimidiata & $\begin{array}{l}\text { Tc, Tr, } \\
\text { Tsp }\end{array}$ & $\begin{array}{l}\text { Boavita (41), Campo Hermoso (41), Chiscas (41), Chitaraque } \\
\text { (41), Chivor (41), Covarachía (41), Cubará (41), Guateque (41, } \\
63) \text {, Guayatá (63), La Uvita (41), Miraflores (63), Moniquirá (41), } \\
\text { Fáez (41), Pisba (41), Puerto Boyacá (41), San Eduardo (41), } \\
\text { San Mateo (41), Sátiva Norte (41), Soatá }(41,44,63,76) \text {, } \\
\text { Susacón (41), Sutatenza (41), Tipacoque }(41), \text { Zetaquirá } \\
(40,41,60,72)\end{array}$ \\
\hline & T. maculata & & Cubará $(41)$, Paya $(40,41)$ \\
\hline
\end{tabular}




\begin{tabular}{|c|c|c|c|}
\hline Departamento & Especie & Flagelados & Municipio \\
\hline $\begin{array}{l}\text { Boyacá } \\
\text { (continuación) }\end{array}$ & T. venosa & Tc & $\begin{array}{l}\text { Boavita (41), Campo Hermoso (41), Chinavita (41), Chivor (41), } \\
\text { Garagoa (41), Guateque }(41,63) \text {, Guayatá (41, 63), La Capilla } \\
\text { (41), La Victoria (41), Macanal (41), Moniquirá (41), Munantá, } \\
\text { Pachavita (41), Páez (41), Pisba (41), Ramiriquí, San Pablo de } \\
\text { Borbur (41), Sátiva Norte (41), Soatá (41), Somondoco (41), } \\
\text { Susacón (41), Sutatenza (41), Tenza (41), Tinjacá (63), } \\
\text { Tipacoque (41), Zetaquirá }(40,41,60,72)\end{array}$ \\
\hline Caldas & R. pallescens & $\mathrm{NE}$ & Samaná*. \\
\hline Caquetá & $\begin{array}{l}P . \text { geniculatus } \\
R . \text { pictipes } \\
\text { R. prolixus }\end{array}$ & $\begin{array}{l}\text { Tc } \\
\text { NEG } \\
\text { Tc }\end{array}$ & $\begin{array}{l}\text { Belén de los Andaquíes*, Solano }(62,74,92) \\
\text { Tres Esquinas }(26,63) \\
\text { Puerto Rico*, Santa Hena*, Florencia }(44,63,76) \text {, Maguareño*, } \\
\text { Doncello*. }(60,72)\end{array}$ \\
\hline \multirow[t]{6}{*}{ Casanare } & $\begin{array}{l}\text { C. pilosa } \\
\text { E. mucronatus } \\
\text { P. geniculatus }\end{array}$ & $\begin{array}{l}\mathrm{NE} \\
\mathrm{NE} \\
\mathrm{NE}\end{array}$ & $\begin{array}{l}\text { Nunchia (41), Pore (41), Villanueva (41). (64) } \\
\text { Hato Corozal (41), Sabanalarga (41), Tauramena (41), Villanueva } \\
(41,64)\end{array}$ \\
\hline & P. lignarius & NE & $(64)$ \\
\hline & Ps. arthuri & NE & Paz de Ariporo (41), Monterrey $(41,64)$ \\
\hline & R. prolixus & $\mathrm{Tc}, \mathrm{Tr}$ & $\begin{array}{l}\text { Aguazul (41), Hato Corozal (41), Mani }(41,63,76) \text {, Monterrey } \\
(41) \text {, Nunchia (41), Orocué (41), Paz de Ariporo (41), Pore (41), } \\
\text { Recetor (41), Sabanalarga (41), San Luis de Palenque (41), } \\
\text { Támara (41), Tauramena (41), Trinidad (41), Villanueva (41), } \\
\text { Yopal }(41,60,63,64,72,76)\end{array}$ \\
\hline & T. dimidiata & $\mathrm{Tc}, \mathrm{Tr}$ & Sácama $(41)$, La Salina $(41,60,64,72)$ \\
\hline & T. maculata & Tc, Tsp & $\begin{array}{l}\text { Aguazul (41), Hato Corozal }(26,41) \text {, Monterrey (41), Nunchía } \\
\text { (41), Paz de Ariporo (41), Pore (41), San Luis de Palenque (41), } \\
\text { Tauramena (41), Villanueva }(41) \text {, Yopal }(41,64)\end{array}$ \\
\hline Cauca & P. geniculatus & NE & Gorgona $(60,72,74)$ \\
\hline & $P$. rufotuberculatus & Tc & Santander de Quilichao $(60,72,74)$ \\
\hline Cesar & $\begin{array}{l}\text { R. neivai } \\
\text { R. pallescens } \\
\text { R. prolixus } \\
\text { T. dimidiata } \\
\text { T. maculata }\end{array}$ & $\begin{array}{l}\text { NR } \\
\text { NEG } \\
\text { Tc, Tr } \\
\text { NEG } \\
\text { NR }\end{array}$ & $\begin{array}{l}\text { Valledupar }(60,72) \\
\text { Caserío Los Pajaritos }(26) \\
\text { El Paso (63), Chiriguaná }(63), \text { Río de Oro }(60,63,72) \\
\text { Valledupar }(26) \\
\text { San Juan del Cesar }(34,36,60,72)\end{array}$ \\
\hline Chocó & $\begin{array}{l}\text { P. geniculatus } \\
\text { R. pallescens } \\
\text { T. dispar } \\
\text { T. venosa }\end{array}$ & $\begin{array}{l}\mathrm{NR} \\
\mathrm{TC} \\
\mathrm{NE} \\
\mathrm{NR}\end{array}$ & $\begin{array}{l}\text { Quibdó }(44,63,75) \\
(89) \\
\text { San José del Palmar }(74)\end{array}$ \\
\hline Córdoba & $\begin{array}{l}\text { E. cuspidatus } \\
\text { P. geniculatus } \\
\text { R. pallescens }\end{array}$ & Tc & $\begin{array}{l}(32) \\
(32) \\
(32)\end{array}$ \\
\hline \multirow[t]{7}{*}{ Cundinamarca } & $\begin{array}{l}\text { C. pilosa } \\
\text { E. mucronatus } \\
\text { P. geniculatus }\end{array}$ & $\begin{array}{l}\text { Tc } \\
\mathrm{NE} \\
\mathrm{NE}\end{array}$ & $\begin{array}{l}\text { Girardot (63), Tocaima (63), Villeta }(60,63,72) \\
\text { Medina (41) } \\
\text { Agua de Dios (41), Caparrapi (41), La Mesa (41), La Palma } \\
\text { (41), Medina (41), Nilo (41), Pacho (41), Paime (41), } \\
\text { Paratebueno (41), San Antonio del Tequendama (41), Tibacuy } \\
\text { (41), Tocaima (41), Viotá (41), Yacopi }(41,60,72)\end{array}$ \\
\hline & P. lignarius & $\mathrm{NE}$ & ** \\
\hline & P. rufotuberculatus & $\mathrm{NE}$ & Guayabetal (41), Pacho (41) \\
\hline & R. colombiensis & NE & Nilo $^{\star}$, Apulo* \\
\hline & R. pallescens & NE & Yacopí (41) \\
\hline & R. pictipes & NE & Medina (42) \\
\hline & R. prolixus & $\mathrm{Tc}, \mathrm{Tr}$ & $\begin{array}{l}\text { Agua de Dios (41), Anapoima }(41,44,63) \text {, Anolaima }(44,63,76) \text {, } \\
\text { Apulo }(44,63,76) \text {, Cáqueza }(44,63) \text {, Choachi }(44,63,76) \text {, } \\
\text { Fómeque }(44,63,76) \text {, Fosca }(41) \text {, Fusagasugá }(44,63,76) \text {, } \\
\text { Gachalá }(41,63) \text {, Gachetá }(44,63) \text {, Girardot }(44,63,76) \text {, }\end{array}$ \\
\hline
\end{tabular}




\begin{tabular}{|c|c|c|c|}
\hline Departamento & Especie & Flagelados & Municipio \\
\hline \multirow[t]{4}{*}{ Cundinamarca } & $\begin{array}{l}\text { R. prolixus } \\
\text { (continuación) }\end{array}$ & $\mathrm{Tc}, \mathrm{Tr}$ & $\begin{array}{l}\text { Guachetá (63), Guaduas }(63,76) \text {, Guayabal }(63) \text {, La Mesa }(41, \\
63,79), \text { La Palma }(63,76,79) \text {, La Unión }(44,63,76) \text {, La Vega } \\
(44,63,76) \text {, Machetá }(44,63) \text {, Manta }(44,63,76) \text {, Medina }(41), \\
\text { Mesitas del Colegio }(44,63) \text {, Nariño }(41,63,76) \text {, Nilo }(41,44,63, \\
\text { 76), Pacho }(41,44,63,76,77) \text {, Pandi }(63) \text {, Paratebueno }(41), \\
\text { Peñón (41), Puerto Salgar }(63,76) \text {, San Antonio de Tena }(63,76 \text {, } \\
\text { 79), San Antonio del Tequendama (41), Tibacuy }(41) \text {, Tibiritá }(44, \\
63,76) \text {, Tocaima }(41,63,76) \text {, Ubalá }(41) \text {, Ubaque }(63,76,79), \\
\text { Villeta (63), Viotá }(44,63,76) \text {, Yacopi }(41,60,72)\end{array}$ \\
\hline & R. robustus & NE & Viotá (42) \\
\hline & T. dimidiata & NEG & Guachetá (63), Mahetá $(60,63,72)$ \\
\hline & T. venosa & $\mathrm{NE}$ & Manta (41), Paime (41), Peñón (41), Tibirita (41), Villagómez (41) \\
\hline \multirow[t]{3}{*}{ Guainía } & P. geniculatus & $\mathrm{NR}$ & Barranco Mina*** Inírida*** \\
\hline & $R$. brethesi & Tc & Cacahual (43), Inírida (43), Puerto Colombia (43) \\
\hline & R. prolixus & NR & Barranco Mina ${ }^{\star \star \star}$ \\
\hline \multirow[t]{4}{*}{ Huila } & P. geniculatus & NE & La Plata $(60,63,72,74)$ \\
\hline & R. prolixus & $\mathrm{Tc}, \mathrm{Tr}$ & $\begin{array}{l}\text { Altamira (63), Baraya }(44,63) \text {, Campoalegre }(44,63) \text {, El Hobo } \\
(44,63) \text {, Garzón }(63) \text {, Gigante }(44,63,76) \text {, Neiva }(44,63,76) \text {, } \\
\text { Planadas }(60,63,72)\end{array}$ \\
\hline & T. dimidiata & Tc & Altamira (63), Garzón $(44,63)$, Neiva $(60,63,72)$ \\
\hline & T. dispar & NE & La Plata (74) \\
\hline \multirow[t]{6}{*}{ La Guajira } & E. cuspidatus & $N R$ & $(36)$ \\
\hline & P. geniculatus & NR & Maicao (36) \\
\hline & R. neivai & $\mathrm{NR}$ & Maicao (36) \\
\hline & R. prolixus & Tc & Manaure $(76,79)$, Riohacha $(60,63,72,76)$ \\
\hline & T. dimidiata & $\operatorname{Tr}$ & Perijá (26) \\
\hline & T. maculata & $\mathrm{NR}$ & Maicao (36), Uribia $(34,36,60,63,72)$ \\
\hline \multirow[t]{6}{*}{ Magdalena } & E. cuspidatus & NE & Guamal $^{*}$ \\
\hline & P. geniculatus & NE & Ciénaga*, Guamal $^{*}$ \\
\hline & R. pallescens & $\mathrm{NE}$ & ElBanco*, Guamal* \\
\hline & R. prolixus & $\mathrm{Tc}, \mathrm{Tr}$ & Pivijay $(76,79)$, Santa Marta* $(60,72)$ \\
\hline & T. dimidiata & Tc & Santa Marta $(26,60,63,72)$ \\
\hline & T. maculata & NEG & Santa Marta* (26) \\
\hline \multirow[t]{12}{*}{ Meta } & C. pilosa & Tc, Tsp & El Porvenir (73), Granada $(60,63,72)$ \\
\hline & E. mucronatus & $N E$ & $(60,72)$ \\
\hline & M. trinidadensis & NE & San Martín $(37,60,63,72)$ \\
\hline & P. geniculatus & Tc & El Porvenir (73), La Macarena (46) \\
\hline & $P$. lignarius & Tc & El Porvenir $(34,60,72,73)$ \\
\hline & P. rufotuberculatus & $\mathrm{NR}$ & $(63)$ \\
\hline & Ps. arthuri & NEG & El Porvenir $(34,60,69,73)$ \\
\hline & R. dalessandroi & $\operatorname{Tr}$ & San Martín $(60,68,72)$ \\
\hline & R. pictipes & Tc & $\begin{array}{l}\text { Cumaral }{ }^{*} \text {, La Macarena (46), Lejanías*, San Martín } \\
(37,60,63,72)\end{array}$ \\
\hline & $\begin{array}{l}\text { R. prolixus } \\
\text { Tsp }\end{array}$ & $\mathrm{Tc}, \mathrm{Tr}$, & $\begin{array}{l}\text { Acacias }(63,76) \text {, Cumaral }(63) \text {, El Porvenir }(34,73) \text {, Granada } \\
(63,76) \text {, Guamal }(63,76) \text {, Guape }(63) \text {, Puerto López }(63,76) \text {, } \\
\text { Restrepo }(44,63,76) \text {, San Antonio (63), San Martín }(44,63) \text {, } \\
\text { Villavicencio }(34,44,60,63,72,76)\end{array}$ \\
\hline & T. dimidiata & $N R$ & Cumaral (84), Restrepo (84) \\
\hline & T. maculata & NEG & El Porvenir (73) \\
\hline Nariño & T. dispar & Tc & Inda (65), Tumaco* \\
\hline \multirow[t]{5}{*}{ Norte de Santander } & E. cuspidatus & NR & $(60,72)$ \\
\hline & E. mucronatus & $\mathrm{Tc}, \mathrm{Tr}$ & $(60,63,72)$ \\
\hline & P. geniculatus & Tc & Tibú (76), Villa del Rosario $(74) .(60,63,72)$ \\
\hline & R. pallescens & & $(45)$ \\
\hline & R. pictipes & & (45) \\
\hline
\end{tabular}




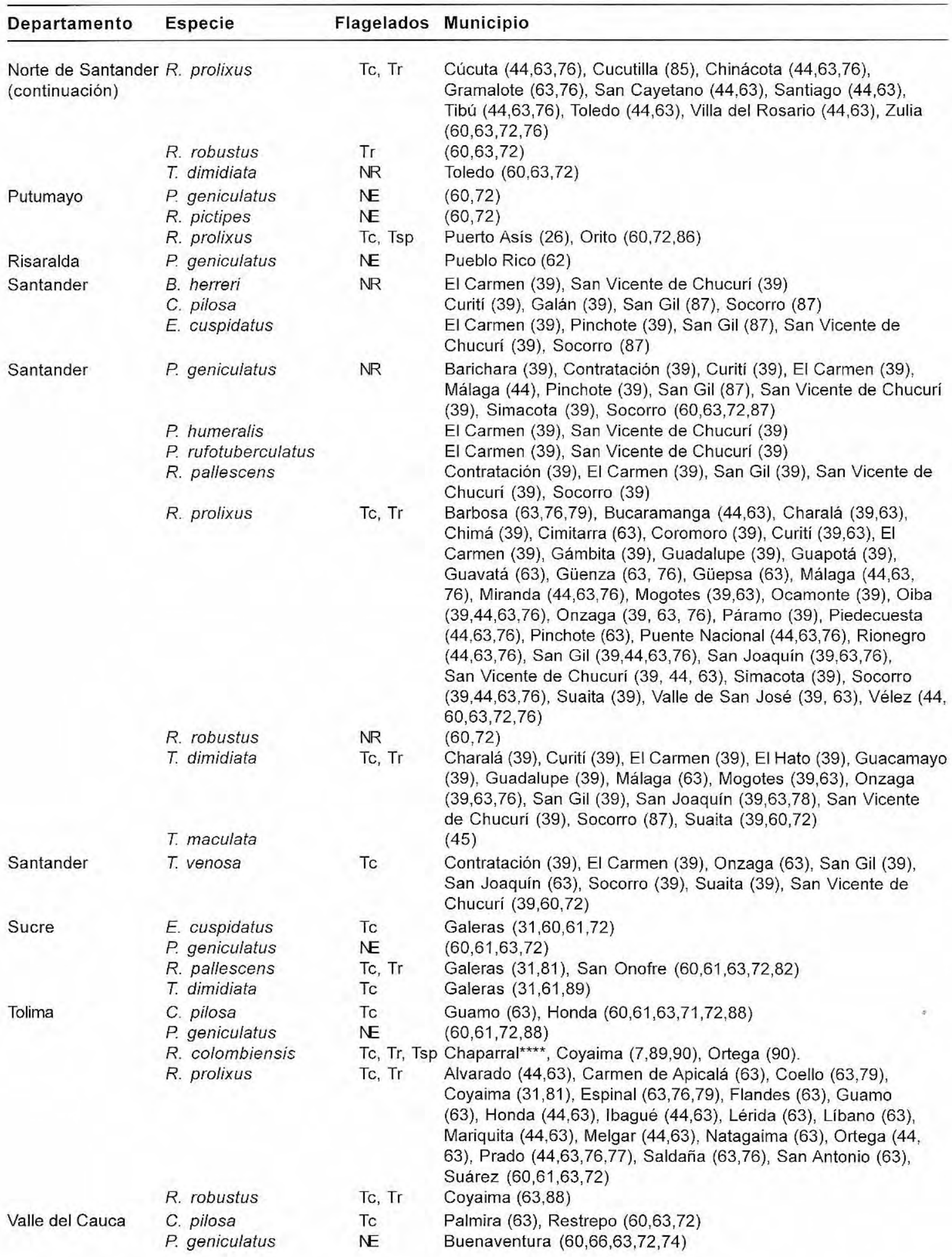




\begin{tabular}{llll}
\hline Departamento & Especie & Flagelados & Municipio \\
\hline \multirow{2}{*}{ Valle del Cauca } & P. rufotuberculatus & $\mathrm{NE}$ & Buenaventura $(60,66,72,74)$ \\
(continuación) & R. prolixus & $\mathrm{NR}$ & Cali $(63)$ \\
& T. dispar & Tc & Buenaventura $(66,74)$, Calima $(62,65,74)$ \\
Vaupés & E. mucronatus & $\mathrm{NE}$ & Mitú \\
& P. geniculatus & $\mathrm{NE}$ & Mitú* \\
& R. pictipes & $\mathrm{NE}$ & Mitú \\
& R. prolixus & $\mathrm{NE}$ & Mitú \\
Vichada & P. lignarius & $\mathrm{NE}$ & $(72)$ \\
& R. prolixus & $\mathrm{NEG}$ & $(60,72)$ \\
& T. maculata & $\mathrm{NE}$ & $(34,60,72)$ \\
\hline
\end{tabular}

NE: no examinado; NR: no reportado; NEG: negativo

Tc: Trypanosoma cruzi; Tr: Trypanosoma rangeli; Tsp: Trypanosoma sp.

* Material identificado en el Laboratorio de Entomología del INS y remitido por los Servicios de Salud.

** Material identificado en el Centro de Investigaciones en Microbiología y Parasitología Tropical de la Universidad de los Andes.

*** Datos de la Secretaría de Salud de Guainía en preparación para ser publicado.

**** Material recolectado e identificado por Luis Gualdrón.

Aquellos registros que no incluyen en las publicaciones el municipio donde fueron recolectados aparecen en la columna "municipio" solamente con el número de referencia correspondiente.

En tres de los departamentos de Colombia, falta por establecer registros de triatominos: Guaviare, Quindío y San Andrés y Providencia.

La única especie no registrada infectada con tripanosomatidos en Colombia y registrada en otros países como positiva es Psammolestes arthuri con Trypanosoma cruzi. Para las especies Panstrongylus humeralis, Belminus herreri y Microtriatoma trinidadensis no hay hasta el momento registro de ejemplares con infecciones naturales en ninguno de los países del continente (93).

Herrán, Chítaga, Pamplona y Pamplonita como los posibles sitios para registrar su presencia, tomando como referencia los registros de altitud informados para Venezuela, donde se observa que la especie se ha ido dispersando siguiendo el curso natural de la cordillera de los Andes.

Triatoma rubrofasciata: se excluye la posibilidad de registrar esta especie en nuestro pais debido a que fue encontrada por Hase en 1932 (13) en puertos de los estados de Aragua y Anzoátegui y nunca más se ha registrado en el país; es importante recordar la fuerte relación que existe entre esta especie y los roedores de los barcos (15). Es probable, entonces, que el registro de esta especie en Venezuela sea consecuencia del comercio marítimo que se efectuaba entre este país y las islas del Caribe donde también se ha establecido su presencia; tal es el caso de Cuba, República Dominicana, Grenada, Guadalupe, Haití y Jamaica, entre otras (1).

\section{Panamá}

Para 1985 (16), se habían registrado 11 especies, todas ellas presentes en nuestro país: Belminus herreri, Cavernicola pilosa, Eratyrus cuspidatus, Microtriatoma trinidadensis, Panstrongylus geniculatus, $P$. humeralis, $P$. rufotuberculatus, Rhodnius pallescens, $R$. prolixus, Triatoma dimidiata y $T$. dispar.

\section{Ecuador}

Para 1985 (17), se habían registrado 16 especies y en 1999 habian aumentado a 18 especies (18): Cavernicola pilosa, Eratyrus cuspidatus, E. mucronatus, Panstrongylus chinai, $P$. geniculatus, $P$. herreri, $P$. howardi, $P$. lignarius, $P$. rufotuberculatus, Rhodnius pictipes, $R$. prolixus, $R$. robustus, $R$. ecuadoriensis, T. carrioni, Triatoma dimidiata, T. dispar, T. infestans y $T$. venosa.

De estas 18 especies, 12 se encuentran en nuestro país y las seis restantes son Panstrongylus chinai, P. herreri, $P$. howardi, Rhodnius ecuadoriensis, Triatoma carrioni y $T$. infestans.

Panstrongylus chinai: la presencia de esta especie ya se analizó en el apartado dedicado a Venezuela. 
Panstrongylus howardi: se considera como una especie con origen en el Ecuador (18) y que hasta el momento se ha encontrado en la provincia costera de Manabí $(17,18)$. Con este único registro es difícil establecer la presencia de esta especie en nuestro país.

Rhodnius ecuadoriensis: especie con presencia en la provincia sureña de Loja y con las costeras de Manabí, Guayas y El Oro y posiblemente en los valles subtropicales de la provincia de Pichincha en Ecuador $(17,18)$ y en el norte de Perú. Si, además, tenemos en cuenta que - según Schofield y Dujardin (19)- esta especie es el producto de una clina norte-sur al occidente de los Andes y representada en su orden por las especies Rhodnius pallescens, $R$. colombiensis y $R$. ecuadoriensis, encontramos que es poco probable que se encuentre en nuestro país.

Triatoma carrioni: especie con presencia exclusiva en Ecuador y el norte de Perú. En Ecuador se ha registrado en las provincias sureñas de Loja, Azuay, Cañar y El Oro y en los valles subtropicales de la provincia de Pichincha $(17,18)$. Es poco probable que esta especie se encuentre en nuestro país.

Triatoma infestans: esta especie tiene registros en las provincias de Esmeraldas e Imbabura, ambas fronterizas con Colombia $(17,18)$; sin embargo, si tenemos en cuenta que estos registros se consideran como dudosos (18), que se trata de una especie que pertenece al complejo Infestans del género Triatoma con distribución exclusiva en el suroriente de Suramérica (15) y, tomando como referencia los éxitos alcanzados en la eliminación de esta especie en los países del cono sur $(20,21)$, es improbable un registro de esta especie en nuestro país.

\section{Perú}

Para 1985 (22), se habían encontrado 15 especies y, en 1989, Fernández-Loyza registra a Hermanlentia matsunoi como Triatoma matsunoi (9), aumentando a 16 las especies de triatominos del Perú: Belminus peruvianus, Cavernicola pilosa, Eratyrus cuspidatus, E. mucronatus, Hermanlentia matsunoi, Microtriatoma trinidadensis, Panstrongylus chinai, $P$. geniculatus, $P$. herreri, $P$. rufotuberculatus, Rhodnius ecuadoriensis, $R$. pictipes, $R$. robustus, Triatoma carrioni, $T$. dimidiata y $T$. infestans.

De estas 16 especies, nueve se comparten con Colombia; de las restantes siete, cuatro se comparten con Ecuador y ya fueron analizadas, las tres restantes son:

Belminus peruvianus: especie con distribución exclusiva en el Perú, en los departamentos de Amazonas y Cajamarca en la frontera con el Ecuador (22). Es poco probable que se encuentre en nuestro país si, además, tenemos en cuenta que se ha localizado en regiones semiáridas (1) no compatibles con las existentes en la zona del sur de Colombia.

Hermanlentia matsunoi: especie descrita para el norte de Perú. La existencia de este único registro no permite establecer su posible presencia en Colombia.

Panstrongylus herreri: especie con presencia exclusiva en el Perú, en los departamentos de Cajamarca, Amazonas y San Martín en la frontera con Ecuador (22). Es poco probable que se encuentre en nuestro país.

\section{Brasil}

Para 1980, Serra y colaboradores encontraron a Alberprosenia malheiroi; en 1995, Lent y colaboradores describieron a Belminus laportei (4); en 1998, Carcavallo y colaboradores registraron a Triatoma jurbergi (6) y con las 45 especies registradas en 1985 (23), el número llega a 48, las cuales son: Alberprosenia malheiroi, Belminus laportei, Cavernicola lenti, C. pilosa, Eratyrus mucronatus, Microtriatoma borbai, $M$. trinidadensis, Panstrongylus diasi, P. geniculatus, $P$. lenti, $P$. lignarius, $P$. lutzi, $P$. megistus, $P$. tupynambai, $P$. rufotuberculatus, Parabelminus carioca, P. yupurucu, Psammolestes tertius, Rhodnius brethesi, $R$. domesticus, $R$. nasutus, $R$. neglectus, $R$. paraensis, $R$. pictipes, $R$. prolixus, $R$. robustus, $R$. stali, Triatoma arthurneivai, T. brasiliensis, T. circummaculata, T. costalimai, T. deanei, T. infestans, T. jurbergi, T. lenti, T. maculata, T. matogrossensis, $T$. melanocephala, T. oliverai, T. petrochiiae, $T$. pseudomaculata, T. rubrofasciata, T. rubrovaria, T. sordida, T. tibiamaculata, T. vitticeps, $T$. williami y T. wygodzinskyi. 
De ellas, once se encuentran en Colombia, ocho se comparten con países del cono sur $(P$. diasi, P. megistus, P. tupynambai, R. stali, $T$. circummaculata, T. infestans, $T$. rubrovaria y $T$. sordida) y una con el Viejo Mundo ( $T$. rubrofasciata).

Por tanto, 28 especies son exclusivas de Brasil: A. malheiroi, B. laportei, C. lenti, M. borbai, P. lenti, P. lutzi, P. carioca, P. yupurucu, Ps. tertius, $R$. domesticus, $R$. nasutus, $R$. neglectus, $R$. paraensis, T. arthurneivai, T. brasiliensis, $T$. costalimai, T. deanei, T. jurbergi, T. lenti, $T$. matogrossensis, T. melanocephala, T. oliverai, T. petrochiiae, T. pseudomaculata, T. tibiamaculata, $T$. vitticeps, $T$. williami y $T$. wygodzinskyi.

Con excepción de A. malheiroi, B. laportei y $C$. lenti, es poco probable que las demás se encuentren en nuestro país, teniendo en cuenta que presentan distribución geográfica en los estados orientales del Brasil, ya que la mayoría de especies pertenecen al género Triatoma (complejo Infestans) $(1,15)$, cuya distribución está limitada al suroriente de Suramérica (15), y por los patrones de domiciliación de los triatominos en Brasil, que se concentra principalmente hacia el oriente debido a la barrera natural que representa la Amazonia (24).

Sobre los ejemplares de la especie $R$. paraensis, se sabe que fueron capturados dos veces en nidos del roedor Echimys chrysurus (1), especie que tiene distribución en Guyanas y base de la Amazonia brasilera, al este de los ríos Negro y Xingu (25). Si esta asociación es específica entre ambas especies, se puede descartar la presencia del triatomino en nuestro país por la distribución geográfica del roedor.

En 1991, Guhl y colaboradores (26) registraron R. neglectus en la Sierra Nevada de Santa Marta y en Puerto Asís, Putumayo; sin embargo, posteriores revisiones del material permitieron comprobar que se trataba de ejemplares pertenecientes a la especie $R$. prolixus y, por tanto, se descarta la presencia de esta especie en nuestro país.

Con respecto a $R$. domesticus, $R$. nasutus, $R$. neglectus y Ps. tertius, Schofield y Dujardin (19) consideran que corresponden a la línea evolutiva que migró hacia el sur a partir de sus ancestros amazónicos, y se internó en el Brasil. Este hecho contribuiría a reforzar la ausencia de estas especies en nuestro país donde se encuentran los descendientes evolutivos de la línea del norte ( $R$. prolixus y PS. arthuri).

Sobre A. malheiroi y B. laportei, la información obtenida no es suficiente como para establecer su posible presencia en Colombia; sin embargo, por haber sido capturadas en el estado amazónico de Pará y por tratarse de géneros asociados a los bosques, es probable que una búsqueda centrada en los departamentos amazónicos de nuestro país permita ubicarlas si su distribución coincide con la Amazonia.

C. lenti se capturó en el estado de Amazonas en el río Uatumá en una cueva con murciélagos de la especie Phyllostomus elongatus (27); aunque su ubicación es cercana a la frontera con los estados de Roraima y Pará, es posible que por tratarse de una especie amazónica se pueda encontrar en nuestro país en los departamentos de esta región. Al igual que en el caso de $C$. pilosa, su búsqueda debe centrarse en las cuevas o huecos de árboles con murciélagos. Se sabe, además, que las cuatro especies del género Phyllostomus presentes en Centro y Suramérica se encuentran por debajo de los 1300 msnm (25), incluida Colombia (28).

\section{Vectores de la enfermedad de Chagas en Colombia}

Un triatomino se considera vector eficiente de la enfermedad de Chagas si reúne los siguientes criterios: 1) estar domiciliado, 2) estar infectado con Trypanosoma cruzi, 3) transmitir el parásito en sus heces y 4) defecar durante el tiempo en que permanece en contacto con la fuente de alimento.

Al aplicar estos criterios a la situación colombiana encontramos que las principales especies domiciliadas son $R$. prolixus, $T$. dimidiata, $T$. maculata y $T$. venosa, y que afectan a los habitantes de 15 departamentos del oriente del país (29). Otras especies que recientemente se han considerado de importancia por sus pruebas de domiciliación, son $P$. geniculatus en el 
municipio de Amalfi (Antioquia) (30); R. pallescens en los departamentos de la Costa Atlántica (3133), R. robustus en Arauca (91) y E. mucronatus en los municipios de Convención, El Carmen y San Cayetano (Norte de Santander) (comunicación personal del ingeniero Florentino Celis de la Secretaría de Salud de Norte de Santander).

Las especies con hábitos silvestres específicos y que, por tanto, no juegan un papel importante en la transmisión de la tripanosomosis americana son $P$ s. arthurien nidos de aves, palmas o debajo de la corteza de árboles muertos $(1,34)$; $P$. lignarius en nidos de aves (34); $C$. pilosa en cuevas o árboles con murciélagos $(1,35) ; B$. rugulosus en palmas (36); $R$. colombiensis en palmas de la especie Attalea butyracea (7); T. disparen zonas boscosas $(1,37)$ y $M$. trinidadensis debajo de la corteza de árboles muertos (1).

De las 23 especies presentes en Colombia, 15 se han encontrado con infecciones naturales de tripanosomátidos identificados como Trypanosoma cruzi (cuadro 2). Entre éstas, se pueden resaltar como posibles vectoras a aquellas especies silvestres que, ocasionalmente, se han localizado intradomiciliarmente por su atracción a la luz artificial (38) y que son: $P$. rufotuberculatus, $E$. cuspidatus, E. mucronatus, $R$. pictipes, $R$. robustus, $R$. dalessandroi, $R$. neivai, $R$. brethesi $(1,36,39-46)$. Las especies capturadas en intra o peridomicilios, pero sin registros de infección con tripanosomas, son $B$. herreri (39) y $P$. humeralis (31).

En cuanto a los tiempos de defecación, se tiene que en $R$. pictipes el $45,5 \%$ de las veces el insecto defeca inmediatamente y en $60 \%$ de los casos ocurre sobre el huésped, principalmente en los estadios de ninfa (47). R. prolixus demora 15,26 minutos en llenarse de sangre y defeca en promedio a los 6,82 minutos después de comenzar a ingerir sangre (48).

La importancia epidemiológica para la transmisión de la enfermedad de Chagas de algunas de las especies de triatominos en Suramérica se presentó en el trabajo de Dias, quien encuentra que los porcentajes de insectos que defecan durante o poco después de la alimentación son los siguientes: $R$. prolixus, $50 \%, R$. neglectus y
T. infestans, 30\%, P. megistus, 22,7\%, T. sordida, $12,5 \%$ y $T$. vitticeps, $0 \%$ (49).

Zeledón y colaboradores (50) presentan un índice de defecación (porcentaje de insectos que defecan dentro de 10 minutos por número promedio de defecciones en 10 minutos/100), según el cual el orden de defecación de tres especies de triatominos es el siguiente: $R$. prolixus, $T$. infestans y $T$. dimidiata.

Pipkin (51) encontró que $96 \%$ de $R$. prolixus defeca dentro de los 30 minutos en comparación con $R$. pallescens, en el cual tan sólo $27 \%$ ha defecado durante el mismo período de tiempo.

Recientemente, factores adicionales específicos como la actividad inmunosupresora celular y humoral que presenta la saliva de $R$. prolixus, pueden contribuir a que se dé alimentación repetida sobre un mismo huésped y, posiblemente, a la transmisión de Trypanosoma cruzi (52).

\section{Discusión}

Tomando como base la distribución de las especies de triatominos presentes en los países vecinos de Colombia, existe la posibilidad de tener en nuestro país representantes de géneros con hábitos silvestres como son Alberprosenia malheiroi, Belminus laportei y Cavernicola lenti, todos ellos en los departamentos de la Amazonia; Triatoma nigromaculata en la Cordillera Oriental y con hábitos intradomiciliarios; Panstrongylus chinai con posibilidad de encontrarse en hábitats selváticos o en las viviendas de los colonos de la Amazonia debido a su atracción por la luz, y Alberprosenia goyovargasi en hábitats silvestres de la frontera con Venezuela.

Los nuevos registros de triatominos en departamentos amazónicos colombianos como Amazonas (53), Guainía (43) y Vaupés han contribuido a mejorar la visión de la distribución de estos insectos en nuestro país y a resaltar la importancia de la vigilancia epidemiológica de la enfermedad de Chagas en estos departamentos. Es así que para complementar los datos entomológicos se tiene en La Pedrera (Amazonas), el primer registro de un caso de Chagas autóctono en una niña de 4 años (54). Este registro, junto con los 38 casos de enfermedad de Chagas 
registrados hasta 1992 en la Amazonia brasileña (55), contribuyen a aumentar la distribución geográfica de esta enfermedad considerada ausente en humanos en la Amazonia.

El cuadro esbozado anteriormente describe aún más acertadamente la transmisión vectorial de la enfermedad de Chagas en Colombia cuando se le incorporan ingredientes adicionales como la presencia en 21 departamentos de $R$. prolixus, considerada la principal especie transmisora en el país; la detección de 15 especies de triatominos con registros de infección natural con T. cruzi; la fuerte intervención antrópica sobre los ecótopos naturales utilizados por los triatominos silvestres, situación que está determinando un cambio en el comportamiento de estas especies (56) y que puede contribuir a explicar las recientes evidencias de colonización intradomiciliaria por parte de especies consideradas silvestres (30-33,91), y la presencia de especies peridomésticas con posibilidades crecientes de domiciliación.

Adicionalmente, el estudio de los triatominos silvestres está cobrando una importancia cada vez mayor, si se tiene en cuenta que varias de estas especies pueden comenzar a jugar un papel mucho más importante en la epidemiología de la enfermedad de Chagas en aquellos lugares donde las actividades de control están dejando las viviendas rurales como nichos ecológicos estables disponibles, que podrían ser ocupados por ellas $(57,58)$.

Para terminar, es importante resaltar que los nuevos registros de especies de triatominos en Colombia están a la espera de la implementación de un eficiente sistema de vigilancia entomológica que para futuros trabajos centre su interés en las especies silvestres como un elemento fundamental de la epidemiología de la enfermedad de Chagas, al considerarse estos insectos como parte del mantenimiento de la circulación en espiral de los parásitos en la naturaleza (59) y al convertirse en el enlace entre el ciclo silvestre, peridoméstico y doméstico.

\section{Referencias}

1. Lent $\mathbf{H}, \mathbf{W}$ ygodzinsky $\mathbf{P}$. Revision of the Triatominae (Hemiptera, Reduviidae) and their significance as vectors of Chagas disease. Bull Am Mus Nat Hist 1979;163:123520 .
2. Schofield CJ. Triatominae: biología y control. UK: Eurocommunica Publications;1994. p.1-79.

3. Schofield CJ. Biosistemática y evolución de triatomineos. En: Guhl F, Jaramillo $C$, editores. Control de tripanosomosis americana y leishmaniosis: aspectos biológicos, genéticos y moleculares. Santafé de Bogotá: Corcas Editores; 1998. p.42-6.

4. Carcavallo RU, Galindez I, Jurberg J, Galvao C, Lent H. Pictorial keys for tribes, genera and species of the subfamily Triatominae. En: Carcavallo RU, Galindez I, Jurberg $\mathrm{J}$, Lent $\mathrm{H}$, editors. Atlas of Chagas' disease vectors in the Americas. Vol. I. Rio de Janeiro: Editora Fiocruz; 1998. p.107-244.

5. Panzera F. La citogenética como herramienta de estudio de los triatominos. En: Angulo VM, editor. Control y manejo de la tripanosomiasis americana. Bucaramanga: Gráficas Trijaimes; 1999. p.55-62.

6. Carcavallo RU, Galvao C, Lent H. Triatoma jurbergi sp. n. do Norte do Estado do Mato Grosso, Brasil (Hemiptera, Reduviidae, Triatominae) com uma atualizacao das sinonimias e outros taxons. Mem Inst Oswaldo Cruz 1998;93:459-64.

7. Moreno J, Galvao C, Jurberg J. Rhodnius colombiensis sp. n. da Colombia, com quadros comparativos entre estructuras fálicas do genero Rhodnius Stal, 1859 (Hemiptera, Reduviidae, Triatominae). Entomol Vect 1999; 6:601-17.

8. Gorla DE, Dujardin JP, Schofield CJ. Biosystematics of Old World Triatominae. Acta Tropica 1997;63:127-40.

9. Jurberg J, Galvao C. Hermanlentia n. gen. da tribo Triatomini, com um rol de especies de Triatominae (Hemiptera, Reduviidae). Mem Inst Oswaldo Cruz 1997; 92:181-5.

10. Carcavallo RU, Jurberg J, Lent H. Torrealbaia martinezi, GEN NOV. sp.n, da tribo Cavernicolini (Hemiptera, Reduviidae, Triatominae): uma abordagem filogenetica. Entomol Vect 1998; 5:143-50.

11. Lent $\mathbf{H}$, Jurberg J, Galvao C. Revalidacao do Genero Mepraia, Mazza, Gajardo \& Jorg, 1940 (Hemiptera, Reduviidae, Triatominae). Mem Inst Oswaldo Cruz 1994; 89:347-52.

12. Torrealba JW, Tonn RJ, Carcavallo RU. Venezuela. In: Carcavallo RU, Ravinovich JE, Tonn RJ, editores. Factores biológicos y ecológicos de la enfermedad de Chagas. Tomo II. Buenos Aires: Centro Panamericano de Ecología Humana y Salud, Organización Panamericana de la Salud/Organización Mundial de la Salud; 1985. p.46572.

13. Cova Garcia P, Suarez MA. Estudio de los triatomíneos en Venezuela. Publicaciones de la División de Malariologia. Número 11. Caracas: Tipografía Vargas; 1959. p.1-209.

14. Scorza JV, Solarte Y, Moreno E. The epidemiological importance of Triatoma nigromaculata (Stal, 1859) 
colonizing human dwellings of the Venezuelan Andes. Mem Inst Oswaldo Cruz 1994;89:299.

15. Schofield CJ. Biosystematics of the Triatominae. En: Biosystematics of haematophagus insects. Oxford: Ed. M.W. Service; 1988. p.284-312.

16. Curto de Casas SI, Canale DM, Carcavallo RU. América Central. In: Carcavallo RU, Ravinovich JE, Tonn $\mathrm{RJ}$, editores. Factores biológicos y ecológicos de la enfermedad de Chagas. Tomo II. Buenos Aires: Centro Panamericano de Ecología Humana y Salud, Organización Panamericana de la Salud/Organización Mundial de la Salud; 1985. p.339-43

17. Lazo RF. Ecuador. In: Carcavallo RU, Ravinovich JE, Tonn $\mathrm{RJ}$, editores. Factores biológicos y ecológicos de la enfermedad de Chagas. Tomo Il. Buenos Aires: Centro Panamericano de Ecología Humana y Salud, Organización Panamericana de la Salud/Organización Mundial de la Salud; 1985. p.413-27.

18. Aguilar HM, Abad-Franch F, Racines J, Paucar A. Epidemiology of Chagas disease in Ecuador. A brief review. Mem Inst Oswaldo Cruz 1999;94(Suppl.1):38793.

19. Schofield CJ, Dujardin JP. Theories on the evolution of Rhodnius. Actual Biol 1999;21:183-97.

20. TDR. Uruguay declared free of Chagas disease transmission. TDR News 1998;56:6.

21. TDR. Chile and Brazil to be certified free of transmission of Chagas disease. TDR News 1999;59:10.

22. Carcavallo RU, Canale DM. Perú. In: Carcavallo RU, Ravinovich JE, Tonn RJ, editores. Factores biológicos y ecológicos de la enfermedad de Chagas. Tomo II. Buenos Aires: Centro Panamericano de Ecología Humana y Salud, Organización Panamericana de la Salud/Organización Mundial de la Salud; 1985. p.449-55.

23. Espinola HN. Brasil. In: Carcavallo RU, Ravinovich JE, Tonn RJ, editores. Factores biológicos y ecológicos de la enfermedad de Chagas. Tomo II. Buenos Aires: Centro Panamericano de Ecología Humana y Salud, Organización Panamericana de la Salud/Organización Mundial de la Salud; 1985. p.363-72.

24. Forattini OP. Biogeografia, origem e distribuicao da domiciliacao de triatomineos no Brasil. Rev Saude Publ 1980;14:265-99.

25. Emmons LH. Neotropical rainforest mammals. A field guide. Chicago and London: The University of Chicago Press;1990. p.1-295.

26. Guhl F, Marinkelle CJ, Becerra W, Romero C. Nuevos registros de triatomineos en Colombia. Biomédica 1991;11:90-1.

27. Barrett TV, Arias JR. A new triatomine host of Trypanosoma from the central amazon of Brazil: Cavernicola lenti n.sp. (Hemiptera, Reduviidae, Triatominae). Mem Inst Oswaldo Cruz 1985;80:91-6.
28. Muñoz J. Clave de murciélagos vivientes en Colombia. Medellín: Editorial Universidad de Antioquia; 1995. p.1132.

29. Corredor A, Santacruz MM, Páez S, Guatame LA. Distribución de los triatominos domiciliarios en Colombia. Bogotá: Instituto Nacional de Salud; 1990. p.1-144.

30. Wolff M, Castillo D, Arboleda JJ, Uribe J, Peláez C. Panstrongylus geniculatus (Hemipotera: Reduviidae): Evidencias de domiciliación. Resúmenes, XXVI Congreso Sociedad Colombiana de Entomologia, Santafé de Bogotá; 1999. p.70.

31. Moreno J. Recientes estudios epidemiológicos de tripanosomiasis americana en diferentes áreas de Colombia. Biomédica 1991:11:43-4.

32. Restrepo M, Restrepo BN, Salazar CI, Parra GJ. Programa Nacional de Prevención y Control de la Enfermedad de Chagas y la cardiopatía infantil. Nodo occidental: Instituto Colombiano de Medicina Tropical: Antioquia, Córdoba y Bolívar. En: Angulo VM, editor. Control y manejo de la tripanosomiasis americana. Bucaramanga: Gráficas Trijaimes; 1999. p.87-93.

33. Londoño E, Isaza D. Rhodnius pallescens (Barber, 1932) y Trypanosoma cruzi en Urabá. Biomédica 1997; 18:66-7.

34. Barreto M, Barreto P. Aves y pitos en Colombia Cespedesia 1984;13:93-6.

35. Marinkelle CJ. Developmental stages of Trypanosoma cruzi-like flagellates in Cavernicola pilosa. Rev Biol Trop 1982;30:107-11.

36. Morales A, Ferro C, Isaza de Rodríguez C, Cura E. Encuesta sobre artrópodos de interés médico en La Guajira, Colombia, Suramérica. Biomédica 1987;7:8794.

37. D'Alessandro A, Barreto P, Duarte R. Distribution of triatomine-transmitted trypanosomiasis in Colombia and new records of the bugs and infections. J Med Entomol 1971;8:159-72.

38. Zeledon R, Rabinovich JE. Chagas'disease: an ecological appraisal with special emphasis on its insect vectors. Ann Rev Entomol 1981;26:101-33.

39. Angulo VM, Gutiérrez R, Rubio I, Joya M, Arismendi M, Esteban L, et al. Triatomineos domiciliados y silvestres: impacto en la transmisión de la enfermedad de Chagas en Santander. En: Angulo VM, editor. Control y manejo de la tripanosomiasis americana. Bucaramanga: Gráficas Trijaimes; 1999. p.72-6.

40. Pinto N, Molina J, Zipa N, Cuervo R, Guhl F. Determinación de la distribución de triatominos en el departamento de Boyacá. Resúmenes, XXVI Congreso Sociedad Colombiana de Entomología, Santafé de Bogotá; 1999. p.69.

41. Nodo Universidad de los Andes, CIMPAT. Informe final de la primera fase del Proyecto Nacional de 
Prevención y Control de la Enfermedad de Chagas y la Cardiopatía Infantil presentado al Ministerio de Salud. Bogotá: Universidad de los Andes; 1999. p.340.

42. Guhl F, Pinto N, Molina J. Nuevos registros de Rhodnius pictipes y Rhodnius robustus (Reduviidae: Triatominae) en Cundinamarca, Colombia. Biomédica $1997 ; 17: 152$

43. Villegas ME, Manotas LE, Molina J, Guhl F. Primer reporte de la presencia de Rhodnius brethesi Matta, 1919 en Colombia. Resúmenes, XXVI Congreso Sociedad Colombiana de Entomología, Santafé de Bogotá; 1999. p.68.

44. Ucros H. Distribución de los triatominae en Colombia. Rev Fac Med Bogotá 1960;28:181-9.

45. Nodo Santander, CINTROP. Informe final de la primera fase del Proyecto Nacional de Prevención y Control de la Enfermedad de Chagas y la Cardiopatía Infantil presentado al Ministerio de Salud. Bucaramanga: Universidad Industrial de Santander; 1999. p.280.

46. Molina JA, GuhI F, Marinkelle CJ. Primer registro de Rhodnius pictipes y Panstrongylus geniculatus (Reduviidae: Triatominae) en PNN Tinigua, La Macarena, Colombia. Biomédica 1995;15:86.

47. Da Silva Rocha D, Galvao C, Jurberg J. Biologia do Rhodnius pictipes Stal, 1872 em condicoes de laboratorio (Hemiptera, Reduviidae, Triatominae). Mem Inst Oswaldo Cruz 1994;89:265-70.

48. Kirk ML, Schofield CJ. Density-dependent timing of defecation by Rhodnius prolixus, and its implications for the transmission of Trypanosoma cruzi. Trans Roy Soc Trop Med Hyg 1987;81:348-9.

49. Dias E. Observacoes sobre eliminnacao de dejecoes e tempo de succao en alguns triatomineos sul-americanos. Mem Inst Oswaldo Cruz 1956;54:115-24

50. Zeledon R, Alvarado R, Jiron LF. Observations on the feeding and defecation patterns of three triatomine species (Hemiptera:Reduviidae). Acta Tropica 1977; 34:65-77.

51. Pipkin AC. Domiciliary reduviid bugs and the epidemiology of Chagas' disease in Panama (Hemiptera:Reduviidae:Triatominae). J Med Entomol 1968;5:107-24.

52. Kalvachova P, Hribalova V, Kodym P, Volf $P$. Modulation of murine lymphocyte responsiveness by the saliva of Rhodnius prolixus (Hemiptera: Reduviidae). J Med Entomol 1999;36:341-4.

53. Gualdrón LE, Brochero HL, Arévalo C, Pérez L, Suárez M, Olano VA. Hallazgo de algunos vectores de la enfermedad de Chagas en el departamento del Amazonas. Resúmenes, XXVI Congreso Sociedad Colombiana de Entomología, Santafé de Bogotá; 1999. p.72.

54. Martinez FE, Blanco AJ, Hernandez SM, Suarez MC, Molina JA, Santacruz MM, et al. First indigenous case of Chagas disease from the province of Amazonas in Colombia. Simposio Internacional sobre Doenca de Chagas, Rio de Janeiro; 1999.

55. Rodrigues J, Verissimo AC, Neves M, Fernandes O. Chagas disease: from bush to huts and houses. Is it the case of the brazilian Amazon? Mem Inst Oswaldo Cruz 1999;94:379-84.

56. Castillo D, Wolff M. Aspectos del comportamiento de los triatominos (Hemiptera: Reduviidae), vectores de la enfermedad de Chagas. Biomédica 2000;20:59-64.

57. Borges EC, Pires HHR, Barbosa SE, Nunes CMS, Pereira MH, Romanha AJ, et al. Genetic variability in brazilian triatomines and the risk of domiciliation. Mem Inst Oswaldo Cruz 1999;94:371-3.

58. Schofield CJ, Diotaiuti L, Dujardin JP. The process of domestication in Triatominae. Mem Inst Oswaldo Cruz 1999;94:375-8.

59. Pavlovsky E. Natural nidality of transmissible diseases with special reference to the landscape epidemiology of zooanthroponoses. Urbana and London: University of Illinois Press; 1966. p.1-261.

60. D'Alessandro A, Barreto P. Colombia. In: Carcavallo $\mathrm{RU}$, Ravinovich JE, Tonn RJ, editores. Factores biológicos y ecológicos de la enfermedad de Chagas. Tomo II. Buenos Aires: Centro Panamericano de Ecología Humana y Salud, Organización Panamericana de la Salud/ Organización Mundial de la Salud; 1985. p.377-99.

61. Moreno J. Estudios epidemiológicos sobre la enfermedad de Chagas en algunas regiones de Colombia. In: Guhl F, editor. Memorias del curso de posgrado, Genética poblacional de triatomineos aplicada al control vectorial de la enfermedad de Chagas. Santafé de Bogotá: Corcas Editores Ltda.; 1997. p.35-41.

62. Barreto M, Burbano ME, Barreto P. Nuevos registros de flebotomineos (Diptera: Psychodidae) y triatominos (Hemiptera: Reduviidae) para Risaralda, Cauca y Valle del Cauca, Colombia. Colombia Médica 1997;28:116-22.

63. Marinkelle CJ. Colombian triatominae and their infestation with trypanosomatid flagellates. Mitt Inst Colombo-Alemán Invest Cient 1972;6:13-29.

64. Grupo de ETV, Secretaría de Salud de Casanare. Vectores de la enfermedad de Chagas en Casanare, Colombia. Yopal: Secretaría de Salud de Casanare; 1997.

65. Loyola E, Freyre JL, Holguín AF, Sánchez A, González A, Barreto M. Trypanosoma cruzi infections in sylvatic hosts on the Pacific coast of Colombia. Trans Roy Soc Trop Med Hyg 1987;81:760.

66. Barreto M, Barreto P. Triatoma dispar (Hemiptera: Reduviidae), a new record for Colombia. J Med Entomol 1984;21:750.

67. Restrepo MI, Morales A, Ferro C. Presencia del Triatoma dispar Letn, 1950. Colombia. Boletín Epidemiológico de Antioquia 1989;1-2:109. 
68. Carcavallo R, Barreto P. Una nueva especie de Rhodnius Stal (Hemiptera, Reduviidae, Triatominae) de Colombia. Bol Dir Malariol Saneamiento Ambiental 1976;16:176-83.

69. Barreto M, Barreto P, D'Alessandro A. Psammolestes arthuri (Hemiptera: Reduviidae) and its parasite Telenomus capito (Hymenoptera: Scelionidae) in Colombia. J Med Entomol 1984;21:703-5.

70. Wolff M, Arboleda JJ, Gonzalez C, Manotas LE, Rueda AM. Estudio tripanosomiasis americana, municipio de Amalfi, vereda Montebello, 1994. Boletín Epidemiológico de Antioquia 1994;3:302-5.

71. Marinkelle CJ. Developmental stages of Trypanosoma cruzi-like flagellates in Cavernicola pilosa. Rev Biol Trop 1982;30:107-11.

72. D'Alessandro A, Barreto P, Thomas M. Nuevos registros de triatominos domiciliarios y extradomiciliarios en Colombia. Colombia Médica 1981;12:75-85.

73. D'Alessandro A, Barreto P, Saravia N, Barreto M. Epidemiología de Trypanosoma cruzi en los Llanos Orientales de Colombia. Colombia Médica 1985;16:8493.

74. Barreto P, Barreto M, Hurtado C. Nuevos hallazgos en Colombia de Panstrongylus geniculatus (Latreille, 1811) y Triatoma dispar Lent, 1950 (Hemiptera: Reduviidae). Colombia Médica 1988;19:64-7.

75. Dunn LH. Notes on some insects and other arthropods affecting man and animals in Colombia. Am J Trop Med 1929;9:493-508.

76. Ucros H, Rocha H, Duque M. Distribución de Triatominae en Colombia. Antioquia Médica 1971;8:707-17.

77. Uribe C. Infección del Rhodnius prolixus Stahl por Tripanosoma cruzi y Tripanosoma rangeli. Biomédica 1996;16:87-92.

78. Marinkelle CJ. Triatoma dimidiata capitata, a natural vector of Trypanosoma rangeli in Colombia. Rev Biol Trop 1968:15:203-5

79. Ucrós H, Escallón Al. Ampliación de la distribución de los Triatominae en Colombia. Universitas Médicas 1967; 47-9

80. Hernández C. Infección natural del Triatoma capitata Usinger 1939 por el Trypanosoma cruzi. Rev Fac Med $1947 ; 15: 465-80$

81. López G, Moreno J. Genetic variability and differentiation between populations of Rhodnius prolixus and $R$. pallescens, vectors of Chagas'disease in Colombia. Mem Inst Oswaldo Cruz 1995:90:353-7.

82. Romaña CA, Pizarro JC, Rodas R, Guilbert E. Palm trees as ecological indicators of risk areas for Chagas disease. Trans Roy Soc Trop Med Hyg 1999:93:594-5.
83. Ospina S. Situación actual de la enfermedad de Chagas. En: OPS/OMS/Ministerio de Salud, Dirección de Campañas Directas. III reunión de investigadores de malaria y otras enfermedades tropicales. Rionegro, Antioquia: Ministerio de Salud; 1991. p.69-70.

84. Castro AT. Soroprevalencia e conditionantes para Doenca de Chagas (Tripanossomiase americana) regiao do sope da Cordilheira Oriental, Estado de Meta, Colombia (tesis). Sao Paulo: Universidad de Sao Paulo; 1998.

85. Duque S, Peláez D, Gualdrón LE, Villarreal E, Corredor A. Aislamiento de tripanosomas a partir de materia fecal de Rhodnius prolixus. Biomédica 1988; $8: 37-9$

86. Ospina S. Estudio preliminar de un foco de tripanosomiasis amerciana en el municipio de Coyaima, departamento de Tolima. En: OPS/OMS/Ministerio de Salud, Dirección de Campañas Directas. III reunión de investigadores de malaria y otras enfermedades tropicales. Rionegro, Antioquia: Mininsterio de Salud; 1991. p.71.

87. Angulo VM, Tarazona Z, Arismendi MJ, Joya MI, Sandoval CM. Distribución de triatomineos (Hemiptera: Reduviidae) domiciliarios en 27 municipios de Santander. Biomédica 1997;17:81.

88. Vallejo G, Jaramillo JC, Silva JC, Castañeda N, Gualtero D, Lozano LE, et al. Implementación de un programa de control de la transmisión vectorial de la enfermedad de Chagas en el municipio de Coyaima, Tolima. Biomédica 1997;17:94-6.

89. Moreno J. Estudio de vectores y reservorios de Trypanosoma cruzi en algunas regiones de Colombia. En: Vallejo GA, Carranza JC, Jaramillo JC, editores Biologia, epidemiología y control de la tripanosomosis americana y leishmaniosis. Ibagué: Lito-Ediciones; 2000. p.19-22.

90. Vallejo GA, Lozano LE, Carranza JC, Sánchez JL, Jaramillo JC, Guhl F, et al. Ecología de los triatominos no domiciliados en Colombia con especial referencia a Rhodnius colombiensis en el departamento del Tolima En: Vallejo GA, Carranza JC, Jaramillo JC, editores. Biología, epidemiología y control de la tripanosomosis americana y leishmaniosis. Ibagué: Lito-Ediciones; 2000. p. 22-8.

91. Gutiérrez R, Angulo VM, Aguilar F, Reyes A, Tarazona Z, Sandoval CM. Aspectos ecológicos de la enfermedad de Chagas en la región nororiental de Colombia. En: Vallejo GA, Carranza JC, Jaramillo JC: editores. Biología, epidemiología y control de la tripanosomosis americana y leishmaniosis. Ibagué: LitoEdiciones; 2000. p.33-5.

92. Minter-Goedbloed E, Minter DM, Cadena A, Howells RE. First record of Trypanosoma (Schizotrypanum) cruzi from western Amazon basin, Caquetá, Colombia. Trans Roy Soc Trop Med Hyg 1987;81:612. 
93. Sherlock IA, Carcavallo RU, Galindez I. List of natural and experimental flagellate infections in several Triatominae species. En: Carcavallo RU, Galindez I, Jurberg J, Lent H, editors. Atlas of Chagas' disease vectors in the Americas. Vol. I. Rio de Janeiro: Editora Fiocruz; 1998. p. 289-98.
94. Guhl F. Estado actual del control de la enfermedad de Chagas en Colombia. En: Guhl F, Jaramillo C, editores.Curso-taller, Control de tripanosomosis americana y leishmaniosis: aspectos biológicos, genéticos y moleculares. Santafé de Bogotá: Corcas Editores; 1998. p.47-81. 